\title{
Face-to-face engineering of ultrathin Pd nanosheets on amorphous carbon nitride for efficient photocatalytic hydrogen production
}

\author{
Yonghua Tang ${ }^{1,2}$, Peng Zhou ${ }^{1}$, Yuguang Chao ${ }^{1}$, Fei Lin ${ }^{1,3}$, Jianping Lai ${ }^{1}$, Hongxing Li $^{2}$ and \\ Shaojun Guo
}

\begin{abstract}
Photocatalytic hydrogen production represents a promising strategy for clean, sustainable, and environmentfriendly energy supply. Up to now, great efforts have been devoted to designing the photocatalysts with noble metal as co-catalyst for visible-light-driven hydrogen evolution, while more efficient photocatalytic systems are still a major challenge. Herein, we report a facile strategy for synthesizing faceto-face ultrathin Pd nanosheets-amorphous carbon nitride (Pd NSs-ACN) structure with large contacting interface and short electronic transmission pathway, which can work as an efficient photocatalyst for hydrogen production. The synthesis starts with the growth of ultrathin Pd NSs, followed by assembly with the visible-light-response ACN through a simple stirring and annealing procedure. The resultant two dimensional face-to-face structures deliver an average hydrogen generation rate of $1.45 \mathrm{mmol} \mathrm{h}^{-1} \mathrm{~g}^{-1}$ at a temperature of $25^{\circ} \mathrm{C}$, almost 2.6 times higher than that of Pd Nps-ACN with particle-to-face structural feature. The efficient photocatalytic activity is ascribed to the formation of high-density of active sites between ultrafine face-to-face contacted Pd NSs and the $\mathrm{ACN}$, which cooperate more synergistically towards photocatalytic hydrogen production. The face-to-face engineered Pd NSs-ACN hybrids also offer a good stability revealed by photocatalytic hydrogen production measurements. The extraordinary performance highlights a powerful engineering model for designing other face-to-face contacting co-catalyst/ photocatalysts, which will be a great impetus to optimize new catalytic transformations.
\end{abstract}

Keywords: face-to-face engineering, ultrathin Pd nanosheets, amorphous carbon nitride, photocatalytic hydrogen production

\section{INTRODUCTION}

Photocatalytic water splitting to produce hydrogen is a promising means of storing solar energy to addressing the current energy and environmental demands. In past years, various inorganic and organic semiconductors have been developed as photocatalysts for hydrogen evolution driven by solar energy [1-7]. The undesirable recombination of photogenerated electrons-holes and the narrow light absorption range limit the efficiency of photocatalytic reactions and further application. To overcome these problems, many strategies have been developed, for instance, morphology control, element doping, metal deposition, and heterostructure formation in the aim of improving the performance of photocatalytic hydrogen production [8-10]. As well known, noble metals (Pt [11-12], Au [13-15], Pd [13] and Ag [16]) can not only trap the photogenerated electrons from the semiconductor to promote the electron-hole separation, but also serve as the catalytic active sites in the adsorption and reduction of $\mathrm{H}^{+}$. Currently, most of hydrogen production systems reported are noble metalloaded photocatalysts.

Much work so far has focused on metal-free polymeric graphitic carbon nitride $\left(\mathrm{g}-\mathrm{C}_{3} \mathrm{~N}_{4}\right)$ as photocatalyst, and transition metal as co-catalyst for photocatalytic hydrogen production, such as $\mathrm{Fe}$ [17], Co [18], Ni [19], $\mathrm{Cu}$ $[20,21]$. However, these noble-metal free/semiconductor systems suffer from a high recombination rate of photoexcited charge carriers, resulting in low photocatalytic activity. At this point, the noble metal/semiconductor photocatalyst systems, with their superior performance, is still the most likely to be industrialized in the future

\footnotetext{
${ }^{1}$ Department of Materials Science and Engineering, and BIC-ESAT College of Engineering, Peking University, Beijing 100871, China

${ }^{2}$ School of Physics and Optoelectronic Engineering, Xiangtan University, Xiangtan 411105, China

${ }^{3}$ School of Materials Science and Engineering, Ocean University of China, Qingdao 266100, China

* Corresponding author (email: guosj@pku.edu.cn)
} 
$[17,21]$. At present, it is necessary to design a noble metalco-catalyst/semiconductor photocatalytic system with high photoelectron mobility for efficiently catalyzing photocatalytic hydrogen production or photocatalytic oxygen production. For all of noble metal/semiconductor systems, the contact model between the noble metal and photocatalyst is particle-to-face [22-24]. Noble metal nanoparticles (NPs) can only provide very limited active sites and slow kinetics, leading to the low photocatalytic efficiency. Inspired by these challenging issues, the development of new methods for engineering noble metal co-catalyst, like nanostructured engineering [13,25], with maximum activity, stability, and minimum costs has become very important. Among various strategies, ultrathin two dimensional (2D) sheet-like structures with a single or few atoms thickness are of great interest due to their small lateral size, high electron mobility, and relatively high surface energy, which is a result of their high surface to volume ratio and high density of unsaturated atoms. Also, ultrathin 2D nanosheets (NSs) with most of atoms exposed for modifications can act as an ideal platform for engineering their properties [26-28].

Herein, we demonstrate a face-to-face engineering fabrication of ultrathin Pd NSs-amorphous carbon nitride (Pd NSs-ACN) structure for photocatalytic hydrogen evolution. In this face-to-face hybrid structure, the ACN serves as light absorber for wide solar spectra harvesting (about below $672 \mathrm{~nm}$ ), and the ultrathin Pd NSs act as an electron transfer relay at the photocatalyst/reaction solution interface to accelerate photoelectron output from photocatalyst to co-catalyst for fast photocatalytic $\mathrm{H}_{2}$ production. The Pd NSs with thickness about $1.0 \mathrm{~nm}$ not only improves the separation of photoelectrons and holes, but also provides more active sites for photocatalytic reactions, which boosts rapid adsorption of hydrogen ions from water and further reduces them into the hydrogen gas. The optimal designed face-to-face Pd NSs-ACN shows almost 2.6 times higher hydrogen evolution activity under visible light than that of Pd NPs-ACN hybrid with particle-to-face structural feature. The ultrathin $\mathrm{Pd}$ NSs loaded on ACN for photocatalytic $\mathrm{H}_{2}$ production has not been explored so far. Our work represents a new interface engineering strategy for the creation of more efficient interface between co-catalyst and photocatalyst for photocatalytic $\mathrm{H}_{2}$ production driven by sunlight.

\section{EXPERIMENTAL SECTION}

Materials

Pd(II) acetylacetonate, poly(vinylpyrrolidone) (PVP), and
$\mathrm{W}(\mathrm{CO})_{6}$ were purchased from Aladdin Ltd. Citric acid (CA), N,N-dimethylformamide (DMF) and cetyltrimethyl ammonium bromide (CTAB) were obtained from SigmaAldrich. All chemicals were used as received without further purification. The water used throughout all experiments was purified through a Millipore system.

\section{Preparation of ACN}

The graphitic carbon nitride (GCN) photocatalyst was prepared according to the literature [29]. $5 \mathrm{~g}$ Melamine was heated at $500^{\circ} \mathrm{C}$ in a tube furnace under an air atmosphere for $4 \mathrm{~h}$, with the ramping rate of $2^{\circ} \mathrm{C} \mathrm{min}^{-1}$, and then cooled to room temperature and ground to get GCN powder. The as-obtained GCN powder was heated at $620^{\circ} \mathrm{C}$ for $2 \mathrm{~h}$ under $\mathrm{N}_{2}$ to get the $\mathrm{ACN}$.

\section{Preparation of Pd NSs}

Pd NSs were synthesized according to the reported method [30]. In typical procedure, $\mathrm{Pd}(\mathrm{II})$ acetylacetonate $\left(\mathrm{Pd}(\mathrm{acac})_{2}, 8 \mathrm{mg}\right), \operatorname{PVP}\left(M_{\mathrm{W}}=24,000,15 \mathrm{mg}\right)$, CA ( $75 \mathrm{mg}), \mathrm{DMF}(5 \mathrm{~mL})$ and CTAB $(30 \mathrm{mg})$ were mixed in a $25 \mathrm{~mL}$ flask and stirred for $1 \mathrm{~h}$ under $\mathrm{N}_{2}$. When the color of the mixture changed from yellow to homogeneous orange-red, $50 \mathrm{mg}$ of $\mathrm{W}(\mathrm{CO})_{6}$ was added into the flask. Then, the flask was sealed and heated at $80^{\circ} \mathrm{C}$ for $1 \mathrm{~h}$. Then the dark blue products were separated by centrifugation using a sufficient amount of acetone, and then washed with ethanol for several times. Finally, the Pd NSs were dispersed in $10 \mathrm{~mL}$ ethanol for later experiment.

\section{Preparation of Pd NSs-ACN}

Firstly, a certain amount of Pd NSs in ethanol solution and ACN (120 mg) were dispersed in $100 \mathrm{~mL}$ ethanol by sonication for $1 \mathrm{~h}$, respectively. Next, the Pd NSs ethanol solution was added into ACN under stirring for $1 \mathrm{~h}$, and then dried under vacuum at $50^{\circ} \mathrm{C}$ for $10 \mathrm{~h}$. Finally, the sample was kept at $200^{\circ} \mathrm{C}$ for $1 \mathrm{~h}$ in a digital-type temperature-controlled oven and then naturally cooled to room temperature. To obtain different weight percent of Pd $0.05 \%, 0.12 \%, 0.36 \%$, and $0.49 \%$ by the inductively coupled plasma mass spectrometry (ICP-MS), the amount of Pd NSs added was $0.5,1.5,3$, and $5 \mathrm{~mL}$, respectively.

\section{Photocatalytic hydrogen production}

The photocatalytic reactions of the composite photocatalysts were carried out in an outer irradiation-type photoreactor (glass) connected to a closed gas-airtight and a constant temperature system. $20 \mathrm{mg}$ of the photocatalysts was dispersed in $90 \mathrm{~mL}$ ultrapure water and 
$10 \mathrm{~mL}$ triethanolamine (TEOA) with sonication. The suspension was thoroughly degassed to remove air and irradiated using a $300 \mathrm{~W}$ Xe-lamp $(\lambda>420 \mathrm{~nm})$. The photocatalytic $\mathrm{H}_{2}$ evolution rate was analyzed using an online Agilent 7890B gas chromatograph (GC, TCD detector, $5 \AA$ molecular sieve columns and Ar carrier). The controlled photo-deposition of $0.36 \mathrm{wt} \% \mathrm{Pd}$ NPs as a reducing co-catalyst in pure $\mathrm{ACN}$ was prepared by dissolving $\mathrm{K}_{2} \mathrm{PdCl}_{4}$ in the reaction solution. All the photocatalytic hydrogen reaction underwent around $25^{\circ} \mathrm{C}$.

\section{RESULTS AND DISCUSSION}

The face-to-face engineering fabrication of Pd NSs-ACN structure chooses ultrathin Pd NSs as a co-catalyst model, which was prepared through a classical method according to the previous literature [30]. The lateral size of the asprepared Pd NSs is about $10.5 \mathrm{~nm}$ (Fig. S1a and b), with an average thickness of about $1.0 \mathrm{~nm}$ (Fig. 1a). The percentage of top and bottom area to the whole surface is up to $90 \%$. The large flat surface of the Pd NSs endows them with an ultrahigh specific surface area, making them highly favorable for surface active applications. Meanwhile, ACN has super-high specific surface area about $252.4 \mathrm{~m}^{2} \mathrm{~g}^{-1}$ (Fig. S2), which is conducive to the immobilization of Pd NSs. These advantages will lead to excellent catalytic performance in photocatalytic system of interface engineering between Pd NSs and ACN semiconductor.
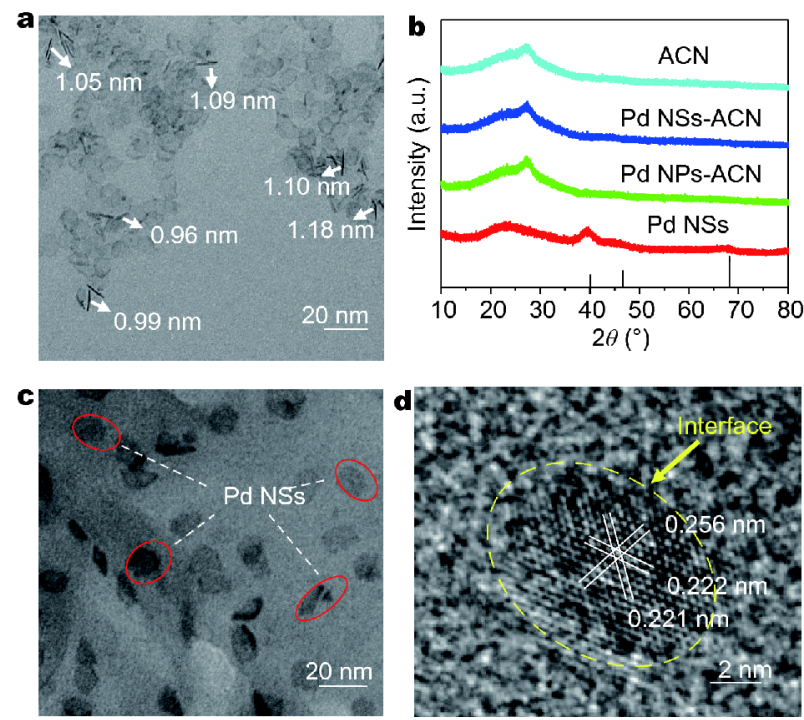

Figure 1 Transmission electron microscopy (TEM) images of Pd NSs (a) and Pd NSs-ACN (c). (b) XRD patterns of the as-synthesized photocatalysts: ACN, Pd NSs-ACN, Pd NPs-ACN and Pd NSs. (d) High resolution TEM (HRTEM) image of the Pd NSs-ACN.
According to the X-ray diffraction (XRD) patterns (Fig. $1 b)$, the Pd NSs-ACN, Pd NPs-ACN and ACN exhibit very similar profiles, where the characteristic peaks located at $27.2^{\circ}$ can be well assigned to the (002) planes of GCN $[29,31]$, due to the accumulation peak of the conjugated aromatic ring. The as-obtained Pd NSs are in the cubic structure (JCPDS 46-1043) [32]. The Pd-ACN did not show the apparent peak of Pd, due to its low Pd content (Fig. S3a). For GCN, two typical diffraction peaks at around $13.1^{\circ}$ and $27.2^{\circ}$ were sharp and intense, indicating its highly crystalline nature (Fig. S3b). However, these two sharp peaks disappear in the ACN pattern, and only one very near at $27.2^{\circ}$ (Fig. $1 \mathrm{~b}$ ), suggesting the absence of long-range order in the atomic arrangements in ACN [33].

As shown in Fig. S4a, many pores with a relatively irregular pore size were formed by heating $\mathrm{GCN}$ in $\mathrm{N}_{2}$, which can provide higher specific surface area. In addition, the high temperature treatment breaks the hydrogen bonds of layered carbon nitride, increasing visible light absorption range by creating abundant band tails. As indicated in Fig. 1c and Fig. S4b, both samples of Pd NSs and NPs have uniform dispersion on the surface of ACN nanosheet to form different types of Pd-ACN hybrid structures. It could be clearly seen that the Pd NPs and ACN contact with each other through the mode of particle-to-face. However, the Pd NSs and ACN contact with each other by another mode of face-to-face, in which the large interfacial areas formed between Pd NSs and ACN guarantee the smooth electron flow from ACN to Pd. The loading of Pd NSs on ACN can be tuned by changing the amount of Pd NSs-ethanol solution added (Fig. S5a-d). The high-resolution TEM (HRTEM) is further carried out to characterize the interface between Pd NSs and ACN (Fig. 1d). From Fig. 1d, the Pd NSs loaded on the surface of ACN exhibit an intimate contact between Pd NSs and ACN, forming the ultrafine Pd NSs-ACN interface. Fig. 1d reveals that the fringes with lattice spacings of 0.256 and $0.22 \mathrm{~nm}$ can be indexed to the Pd $\{200\}$ and $\{111\}$ [30], respectively. Note that the lattice fringes of ACN cannot be resolved by HRTEM, possibly due to its rapid degradation under irradiation of an electron beam.

The chemical states of carbon, nitrogen and palladium in the products (Pd NSs and Pd NPs (0.36\%)-ACN) were investigated by X-ray photoelectron spectroscopy (XPS). The survey XPS spectrum (Fig. 2a) shows the C, N, Pd and $\mathrm{O}$ peaks in Pd-ACN. In Fig. $2 \mathrm{~b}$, the $\mathrm{C}$ 1s peak can be deconvoluted into three peaks at 288.1, 285.2 and $286.5 \mathrm{eV}$, corresponding to $\mathrm{sp}^{2}$-bonded aromatic struc- 

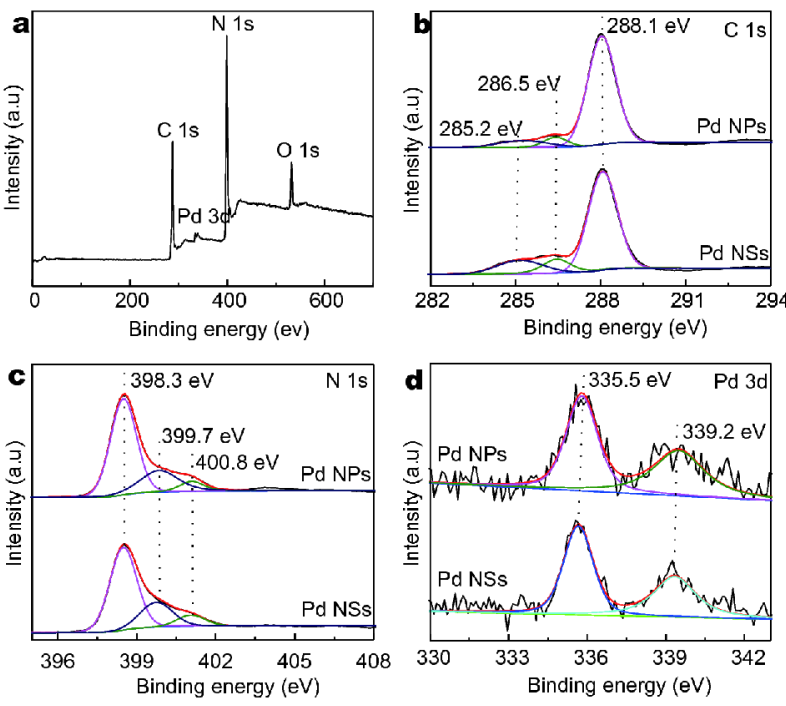

Figure 2 XPS spectra of Pd NPs-ACN and Pd NSs-ACN. (a) Survey spectrum, (b) C 1s, (c) N 1s and (d) Pd 3d high-resolution spectra.

ture $(\mathrm{N}-\mathrm{C}=\mathrm{N})$, graphitc carbon $(\mathrm{C}-\mathrm{C})$, and residual $\mathrm{C}-\mathrm{O}$, respectively. Meanwhile, high-resolution spectrum of $\mathrm{N}$ 1s (Fig. 2c) can also be divided into three peaks at $398.3,399.7$ and $400.8 \mathrm{eV}$, which are associated with nitrogen species in the $\mathrm{N}(\mathrm{C})_{2}, \mathrm{~N}(\mathrm{C})_{3}$, and $\mathrm{N}(\mathrm{CH})$ groups of melon [34], respectively. The two peaks of Pd 3d (Fig. 2d) located at 335.5 and $339.2 \mathrm{eV}$ are assigned to $\mathrm{Pd} 3 \mathrm{~d}_{5 / 2}$ and $\mathrm{Pd} 3 \mathrm{~d}_{3 / 2}$, respectively, which are in good agreement with the metal Pd. Hence, Pd NSs and Pd NPs belong to metal Pd [35].

The photocatalytic activity of all samples was evaluated by the $\mathrm{H}_{2}$ evolution under Xe lamp irradiation $(\lambda$ $>420 \mathrm{~nm}$ ), using TEOA as a scavenger. Fig. 3 a shows the photocatalytic $\mathrm{H}_{2}$-production activity of Pd NSs-ACN (0.36 wt\%), Pd NPs-ACN (0.36 wt\%) and ACN catalysts. For $\mathrm{ACN}$, the rate of $\mathrm{H}_{2}$-production can be almost ignored because of the rapid recombination of photogenerated electron-holes and the quick reversible reaction (hydrogen and oxygen recombination into water). A small amount of Pd loading on ACN can obviously enhance the photocatalytic $\mathrm{H}_{2}$ production. The average hydrogen generation rate of Pd NSs-ACN is $1.45 \mathrm{mmol} \mathrm{h}^{-1} \mathrm{~g}^{-1}$, higher than that of the Pd NPs-ACN $\left(0.56 \mathrm{mmol} \mathrm{h}^{-1} \mathrm{~g}^{-1}\right)$. The enhancement of $\mathrm{H}_{2}$ production of ACN by ultrathin Pd NSs loading is attributed to the following three reasons: 1) the face-to-face contact between the ultrathin Pd NSs and ACN shortens the distance of photoelectron transport from ACN to Pd cocatalyst and inhibits the recombination of photogenerated electrons and holes; 2) the ultrathin Pd NSs provides a
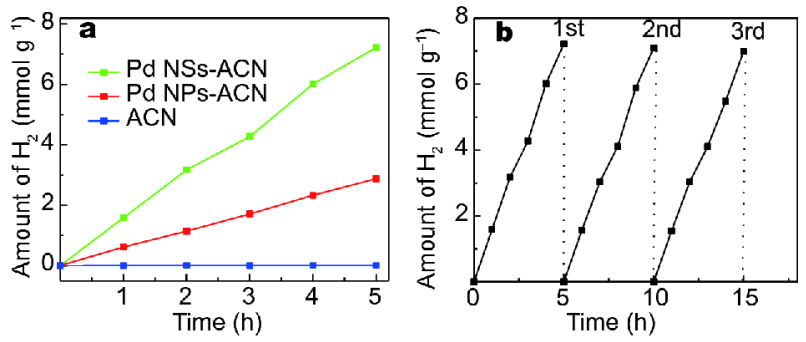

Figure 3 (a) Photocatalytic $\mathrm{H}_{2}$ evolution from the Pd NSs-ACN, Pd NPs-ACN and ACN. (b) Cycle stability test on Pd NSs-ACN photocatalytic $\mathrm{H}_{2}$ evolution under visible light irradiation.

large number of surface active sites capturing the $\mathrm{H}^{+}$from the reaction solution for photocatalytic hydrogen production; 3) a large number of surface atoms exposed by ultrathin Pd NSs serve as an ideal platform for releasing hydrogen gas and further improve the photocatalytic performance. A series of control experiments and photocatalytic tests show the best weight percent (wt\%) of $\mathrm{Pd}$ NSs to ACN is $\sim 0.36 \%$ (Fig. S6).

In addition, to evaluate the stability of the Pd NSsACN, the recycling experiments for the $\mathrm{H}_{2}$ evolution were conducted. The photocatalytic reaction was allowed to process for $1 \mathrm{~h}$ with intermittent evacuation every $5 \mathrm{~h}$ under visible light irradiation $(\lambda>420 \mathrm{~nm})$. As can be seen from Fig. 3b, the $\mathrm{H}_{2}$ evolution rate remains fairly stable after $15 \mathrm{~h}$ irradiation, indicating the long-term photocatalytic stability of $\mathrm{H}_{2}$ evolution by the face-to-face engineered Pd NSs-ACN hybrids, consistent with the TEM results of of Pd NSs-ACN after photocatalytic measurements (Fig. S7). It is worth noting that the excellent solar photocatalytic activity was obtained in the presence of very small amounts of co-catalysts (Pd NSs $0.36 \%$ ), which suggests the striking potential of these Pd NSs-ACN complex structure for practical technology application.

To confirm the interface effect induced by the face-toface engineering between ultrathin $\mathrm{Pd} \mathrm{NS}$ and $\mathrm{ACN}$ which is the main factor in enhancing the $\mathrm{H}_{2}$ evolution activity, the UV-vis diffuse reflectance spectra (DRS), photoelectrochemical test, electrochemical impedance spectroscopy (EIS) measurements, photoluminescence (PL) and time-resolved PL (TRPL) spectra were performed. As show in Fig. 4, the UV-vis absorption of all samples shows a clear absorption edge at $400-600 \mathrm{~nm}$, demonstrating that ACN has strong visible light absorbability. Furthermore, the Pd NSs-ACN exhibits a bit of stronger light absorption in the visible range in comparison with the Pd NPs-ACN control sample, due to the relatively strong surface plasmon resonance (SPR) effect of Pd NSs than in the Pd NPs. Given that the electron- 


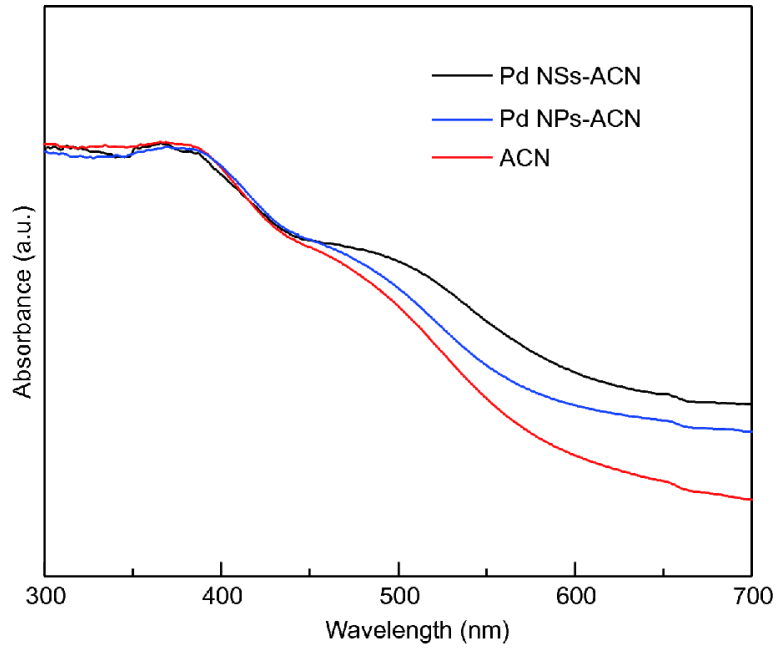

Figure 4 UV-vis DRS of Pd NSs-ACN, Pd NPs-ACN and ACN composites.

hole separation ability can be reflected by the photocurrent, the photoelectrochemical tests were carried out [36]. Fig. 5a reveals that the photocurrents of Pd NSsACN sample are much larger than those of Pd NPs-ACN and ACN, suggesting that Pd NSs can easily trap the electrons from ACN to promote the electron-hole separation in relative to Pd NPs. Moreover, after four lighton and -off cycles, the photoelectrochemical test shows no obvious decay for the Pd NSs-ACN, indicating that the separation and recombination of photogenerated charges maintains constant value.

The effect of face-to-face hybrid structures on the kinetics of interfacial charge immigration was further investigated by EIS analysis. The arc radius on the EIS Nyquist plot (Fig. 5b) reflects the reaction rate on the surface of the electrode. A smaller arc radius corresponds to a more effective separation of photogenerated electronhole pairs and a higher efficiency of charge immigration across the electrode/electrolyte interface. The arc radius of Pd NSs-ACN electrode is smaller than those of Pd NPs-ACN and ACN electrode, suggesting that the Pd NSs-ACN structure can make the separation and immigration of photogenerated electron-hole pairs be more efficient, in good accordance with the result of the photocurrent measurement.

Fluorescence spectroscopy is a powerful tool to determine the transfer and separation efficiency of the photogenerated charge carriers in excited semiconductors. The PL spectra of Pd NSs-ACN and Pd NPsACN excited at $330 \mathrm{~nm}$ are presented in Fig. 5c. Compared with the Pd NPs, the emissions in Pd NSs-ACN
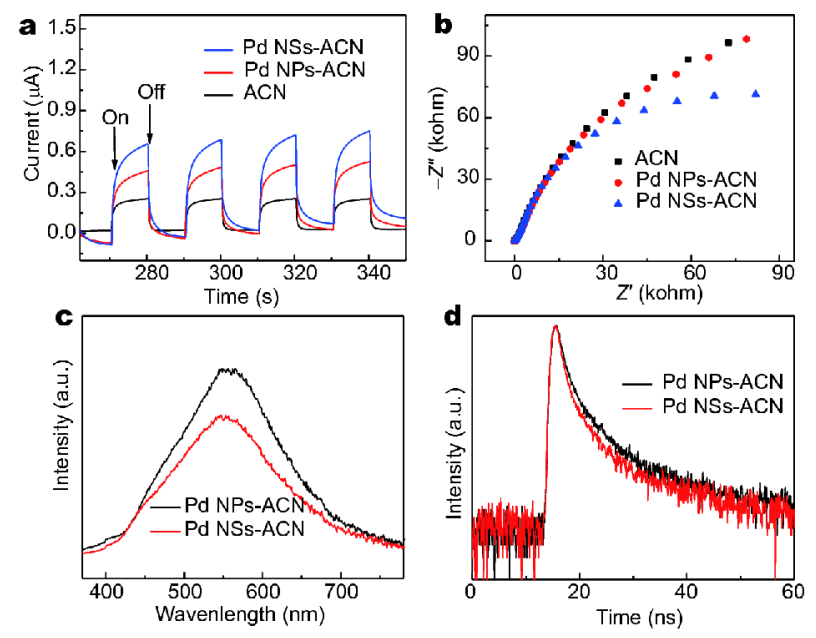

Figure 5 (a) Photocurrent responses of Pd NSs-ACN, Pd NPs-ACN and ACN. (b) EIS Nyquist plots of Pd NSs-ACN, Pd NSs-ACN, and ACN under visible-light conditions. (c) PL spectra of Pd NPs-ACN and Pd NSs-ACN under photoexcitation at $330 \mathrm{~nm}$. (d) The TRPL spectra of Pd NPs-ACN and Pd NSs-ACN monitored under a $404 \mathrm{~nm}$ laser excitation.

were remarkably quenched, suggesting faster transfer of electrons from ACN to Pd NSs, which can suppress the electron-hole recombination and enhance the photocatalytic activity. The PL intensity of Pd NSs-ACN is lower than that of Pd NPs-ACN, which is also consistent with the former $\mathrm{H}_{2}$ evolution activity results [37].

The TRPL spectra (Fig. 5d) monitored at emission peak of each product also prove that the lifetime of Pd NSs$\mathrm{ACN}$ and Pd NPs-ACN is shortened gradually. The decay lifetime of Pd NSs-ACN and Pd NPs-ACN is 8.82 and 11.05 ns (Table 1), respectively. This lifetime can be calculated using the following equation [6]:

$$
<\tau>=\left(f_{1} \tau_{1}^{2}+f_{2} \tau_{2}^{2}+f_{3} \tau_{3}^{2}\right) /\left(f_{1} \tau_{1}+f_{2} \tau_{2}+f_{3} \tau_{3}\right),
$$

where $\tau$ refers to the average lifetime; $\tau_{1}, \tau_{2}$ and $\tau_{3}$ refer to the different excited states of radiative lifetimes; $f_{1}, f_{2}$ and $f_{3}$ refer to the different relative percentages of charge carriers, respectively. The TRPL data further reveal that the lifetime of Pd NSs-ACN is much shorter than that of Pd NPs-ACN. Photocatalysts with co-catalyst materials are expected to have shorter lifetimes due to the efficient charge transfer from the photocatalyst to co-catalyst on the surface and suppression of the electron-hole recombination [38]. All the above results certify that the face-to-face engineering between ultrathin Pd NSs and ACN is more efficient to improve photocatalytic hydrogen evolution.

To understand the drastically enhanced photocatalytic activity of Pd NSs-ACN, we further proposed the photocatalytic mechanism of Pd NSs-ACN and Pd NPs-ACN, 
Table 1 Summary of the photoluminescence decay time $(\tau)$ and their relative amplitude $(f)$ in the Pd NSs-ACN and the Pd NPs-ACN

\begin{tabular}{|c|c|c|c|c|c|c|c|}
\hline \multirow{2}{*}{ Sample } & \multicolumn{3}{|c|}{ Decay time (ns) } & \multicolumn{3}{|c|}{ Relative amplitude (\%) } & \multirow{2}{*}{$\begin{array}{l}\text { Average lifetime } \\
(<\tau>\text {, ns })\end{array}$} \\
\hline & $\tau_{1}$ & $\tau_{2}$ & $\tau_{3}$ & $f_{1}$ & $f_{2}$ & $f_{3}$ & \\
\hline Pd NSs-ACN & 0.75 & 2.77 & 11.79 & 39.36 & 40.81 & 19.83 & 8.82 \\
\hline Pd NPs-ACN & 0.76 & 2.89 & 14.35 & 30.03 & 43.72 & 26.25 & 11.05 \\
\hline
\end{tabular}

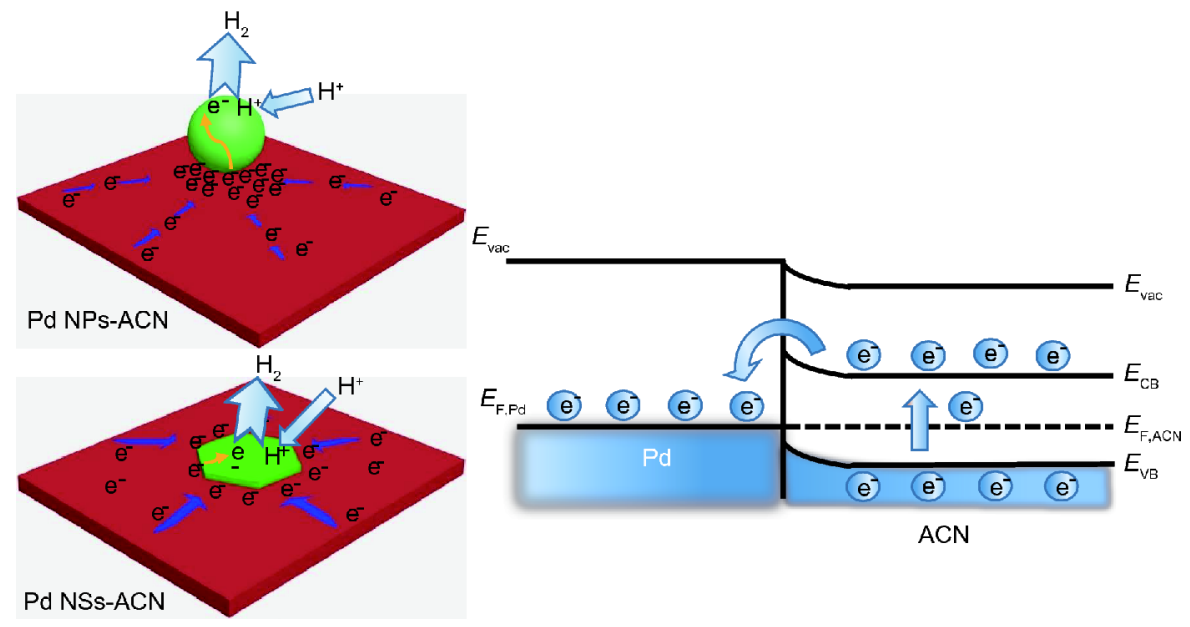

Figure 6 Proposed photocatalytic reaction mechanism of Pd NPs-ACN and Pd NSs-ACN.

respectively. As shown in Fig. 6, electrons in the valence band of the ACN are excited to the conduction band under visible light irradiation, and the photogenerated holes are accumulated at valance band. Because of the lower work function of ACN than Pd [39], the free electrons of ACN could easily transfer to Pd when contacting. It was obvious that ultrathin Pd NSs with lower thickness shorten the distance for electrons to transfer from photo-excited ACN. In addition, the face-to-face contacting in Pd NSs-ACN can provide the direct channel so that the photoelectrons can be transferred to the surface of Pd NSs from ACN for photocatalytic hydrogen reaction. However, due to the limited contact area in particle-to-face Pd NPs-ACN, the photoelectrons are gathered at the touch point and cannot be transmitted to Pd NPs efficiently, which have poor hydrogen production. Besides, the more rapid reduction of $\mathrm{H}^{+}$in Pd NSs$\mathrm{ACN}$ is another key factor for adsorption of new hydrogen ions from the solution after the $\mathrm{H}_{2}$ desorption from the upsides of Pd NSs. Thus, the face-to-face contact between Pd NSs and ACN has more active sites and direct electron channel, revealing excellent photocatalytic performance than in the particle-to-face Pd NPs-ACN.

\section{CONCLUSIONS}

In summary, we demonstrate a feasible approach to synthesize Pd NSs-ACN with distinctive face-to-face structure consisting of large-density active sites and largearea contacting interfaces. These features enable them impressive activity towards photocatalytic hydrogen production. The unique interface in Pd-NSs-ACN shows much superior photocatalytic activities for hydrogen generation than those in the Pd NPs-ACN and ACN. In the face-to-face interface structure, the ultrathin Pd NSs not only promote the rapid transfer of photoelectrons from ACN, but also expose outside surface as an ideal platform for capturing $\mathrm{H}^{+}$to produce $\mathrm{H}_{2}$. In the optimized Pd NSs-ACN (0.36\%) with large contact interface and ultra-short electron transport distance, the hydrogen production activity is over 2.6 times of magnitude than that of particles-to-face Pd NPs-ACN. This work may open a new avenue for photocatalyst discovery and new catalytic optimizer with high solar-driven photocatalytic performance by engineering the interface between the cocatalyst and photocatalyst.

Received 21 May 2018; accepted 20 July 2018;

published online 17 August 2018

$1 \mathrm{Xu}$ J, Yang WM, Huang SJ, et al. CdS core-Au plasmonic satellites nanostructure enhanced photocatalytic hydrogen evolution reaction. Nano Energy, 2018, 49: 363-371

2 Tan CF, Su Su Zin AK, Chen Z, et al. Inverse stellation of CuAu- 
$\mathrm{ZnO}$ multimetallic-semiconductor nanostartube for plasmon-enhanced photocatalysis. ACS Nano, 2018, 12: 4512-4520

3 Wang X, Maeda K, Thomas A, et al. A metal-free polymeric photocatalyst for hydrogen production from water under visible light. Nat Mater, 2009, 8: 76-80

4 Liu G, Yin LC, Wang J, et al. A red anatase $\mathrm{TiO}_{2}$ photocatalyst for solar energy conversion. Energy Environ Sci, 2012, 5: 9603-9610

5 Tang $\mathrm{Y}$, Zhou $\mathrm{P}$, Wang $\mathrm{K}$, et al. BiOCl/ultrathin polyaniline core/ shell nanosheets with a sensitization mechanism for efficient visible-light-driven photocatalysis. Sci China Mater, 2019, 62: 95-102

6 Kang Y, Yang Y, Yin LC, et al. Selective breaking of hydrogen bonds of layered carbon nitride for visible light photocatalysis. Adv Mater, 2016, 28: 6471-6477

7 Wang Z, Wang L. Photoelectrode for water splitting: Materials, fabrication and characterization. Sci China Mater, 2018, 61: 806821

8 Zhou P, Yu J, Jaroniec M. All-solid-state Z-scheme photocatalytic systems. Adv Mater, 2014, 26: 4920-4935

9 Cabán-Acevedo M, Stone ML, Schmidt JR, et al. Efficient hydrogen evolution catalysis using ternary pyrite-type cobalt phosphosulphide. Nat Mater, 2015, 14: 1245-1251

$10 \mathrm{Ge} \mathrm{MZ,} \mathrm{Cao} \mathrm{CY,} \mathrm{Li} \mathrm{SH,} \mathrm{et} \mathrm{al.} \mathrm{In} \mathrm{situ} \mathrm{plasmonic} \mathrm{Ag} \mathrm{nanoparticle}$ anchored $\mathrm{TiO}_{2}$ nanotube arrays as visible-light-driven photocatalysts for enhanced water splitting. Nanoscale, 2016, 8: 52265234

11 Zhang G, Lan ZA, Lin L, et al. Overall water splitting by Pt/g- $\mathrm{C}_{3} \mathrm{~N}_{4}$ photocatalysts without using sacrificial agents. Chem Sci, 2016, 7: 3062-3066

12 Hu C, Han Q, Zhao F, et al. Graphitic $\mathrm{C}_{3} \mathrm{~N}_{4}-\mathrm{Pt}$ nanohybrids supported on a graphene network for highly efficient methanol oxidation. Sci China Mater, 2015, 58: 21-27

13 Shi R, Cao Y, Bao Y, et al. Self-assembled Au/CdSe nanocrystal clusters for plasmon-mediated photocatalytic hydrogen evolution. Adv Mater, 2017, 29: 1700803

14 Liu W, Liu Z, Wang G, et al. Carbon coated $\mathrm{Au} / \mathrm{TiO}_{2}$ mesoporous microspheres: a novel selective photocatalyst. Sci China Mater, 2017, 60: 438-448

15 Zhu Y, Xu Z, Jiang W, et al. Engineering on the edge of Pd nanosheet cocatalysts for enhanced photocatalytic reduction of $\mathrm{CO}_{2}$ to fuels. J Mater Chem A, 2017, 5: 2619-2628

16 Zhu J, Ren J, Huo Y, et al. Nanocrystalline $\mathrm{Fe} / \mathrm{TiO}_{2}$ visible photocatalyst with a mesoporous structure prepared via a nonhydrolytic sol-gel route. J Phys Chem C, 2007, 111: 18965-18969

17 Barakat MA, Schaeffer H, Hayes G, et al. Photocatalytic degradation of 2-chlorophenol by Co-doped $\mathrm{TiO}_{2}$ nanoparticles. Appl Catal B-Environ, 2005, 57: 23-30

18 Bi L, Xu D, Zhang L, et al. Metal Ni-loaded g- $\mathrm{C}_{3} \mathrm{~N}_{4}$ for enhanced photocatalytic $\mathrm{H}_{2}$ evolution activity: the change in surface band bending. Phys Chem Chem Phys, 2015, 17: 29899-29905

19 Fan M, Song C, Chen T, et al. Visible-light-drived high photocatalytic activities of $\mathrm{Cu} / \mathrm{g}-\mathrm{C}_{3} \mathrm{~N}_{4}$ photocatalysts for hydrogen production. RSC Adv, 2016, 6: 34633-34640

20 Qin Z, Wang M, Li R, et al. Novel $\mathrm{Cu}_{3} \mathrm{P} / \mathrm{g}-\mathrm{C}_{3} \mathrm{~N}_{4} \mathrm{p}$-n heterojunction photocatalysts for solar hydrogen generation. Sci China Mater, 2018, 61: 861-868

21 Shiraishi Y, Kofuji Y, Kanazawa S, et al. Platinum nanoparticles strongly associated with graphitic carbon nitride as efficient cocatalysts for photocatalytic hydrogen evolution under visible light. Chem Commun, 2014, 50: 15255-15258

22 Li K, Zeng Z, Yan L, et al. Fabrication of platinum-deposited carbon nitride nanotubes by a one-step solvothermal treatment strategy and their efficient visible-light photocatalytic activity. Appl Catal B-Environ, 2015, 165: 428-437

23 Lacerda AM, Larrosa I, Dunn S. Plasmon enhanced visible light photocatalysis for $\mathrm{TiO}_{2}$ supported Pd nanoparticles. Nanoscale, 2015, 7: 12331-12335

24 Shuang S, Lv R, Xie Z, et al. Surface plasmon enhanced photocatalysis of Au/Pt-decorated $\mathrm{TiO}_{2}$ nanopillar arrays. Sci Rep, 2016, 6: 26670

25 Bai S, Wang X, Hu C, et al. Two-dimensional g- $\mathrm{C}_{3} \mathrm{~N}_{4}$ : an ideal platform for examining facet selectivity of metal co-catalysts in photocatalysis. Chem Commun, 2014, 50: 6094-6097

26 Ge J, Wei P, Wu G, et al. Ultrathin palladium nanomesh for electrocatalysis. Angew Chem Int Ed, 2018, 57: 3435-3438

27 Tan C, Cao X, Wu XJ, et al. Recent advances in ultrathin twodimensional nanomaterials. Chem Rev, 2017, 117: 6225-6331

28 Liu HM, Han SH, Zhao Y, et al. Surfactant-free atomically ultrathin rhodium nanosheet nanoassemblies for efficient nitrogen electroreduction. J Mater Chem A, 2018, 6: 3211-3217

29 Kang Y, Yang Y, Yin LC, et al. An amorphous carbon nitride photocatalyst with greatly extended visible-light-responsive range for photocatalytic hydrogen generation. Adv Mater, 2015, 27: 4572-4577

30 Li Y, Yan Y, Li Y, et al. Size-controlled synthesis of Pd nanosheets for tunable plasmonic properties. CrystEngComm, 2015, 17: 18331838

31 Niu P, Zhang L, Liu G, et al. Graphene-like carbon nitride nanosheets for improved photocatalytic activities. Adv Funct Mater, 2012, 22: 4763-4770

32 Huang X, Tang S, Mu X, et al. Freestanding palladium nanosheets with plasmonic and catalytic properties. Nat Nanotech, 2011, 6: 28-32

33 Yang S, Gong Y, Zhang J, et al. Exfoliated graphitic carbon nitride nanosheets as efficient catalysts for hydrogen evolution under visible light. Adv Mater, 2013, 25: 2452-2456

34 Liu J, Liu Y, Liu N, et al. Metal-free efficient photocatalyst for stable visible water splitting via a two-electron pathway. Science, 2015, 347: 970-974

35 Cheng S, Meng X, Shang N, et al. Pd supported on g- $\mathrm{C}_{3} \mathrm{~N}_{4}$ nanosheets: Mott-Schottky heterojunction catalyst for transfer hydrogenation of nitroarenes using formic acid as hydrogen source. New J Chem, 2018, 42: 1771-1778

36 Ai G, Li H, Liu S, et al. Solar water splitting by $\mathrm{TiO}_{2} / \mathrm{CdS} / \mathrm{Co}-\mathrm{Pi}$ nanowire array photoanode enhanced with $\mathrm{Co}-\mathrm{Pi}$ as hole transfer relay and CdS as light absorber. Adv Funct Mater, 2015, 25: 57065713

37 Xu J, Wang Z, Zhu Y. Enhanced visible-light-driven photocatalytic disinfection performance and organic pollutant degradation activity of porous $\mathrm{g}-\mathrm{C}_{3} \mathrm{~N}_{4}$ nanosheets. ACS Appl Mater Interfaces, 2017, 9: 27727-27735

38 Sun Z, Zheng H, Li J, et al. Extraordinarily efficient photocatalytic hydrogen evolution in water using semiconductor nanorods integrated with crystalline $\mathrm{Ni}_{2} \mathrm{P}$ cocatalysts. Energy Environ Sci, 2015, 8: 2668-2676

39 Tong H, Ouyang S, Bi Y, et al. Nano-photocatalytic materials: Possibilities and challenges. Adv Mater, 2012, 24: 229-251

Acknowledgements This work was financially supported by the National Natural Science Foundation of China (51772255), Hunan Natural Science Foundation (2016JJ3123), the National Key Research and De- 
velopment Program of China (2016YFB0100201) and the start-up supports from Peking University and Young Thousand Talented Program.

Author contributions $\mathrm{Li} \mathrm{H}$ and Guo S designed and engineered this work. Tang Y performed the experiments and wrote the paper with support from Zhou P. All authors contributed to the general discussion.

Conflict of interest These authors declare no conflict of interest.

Supplementary information Experimental data are available in the online version of the paper.

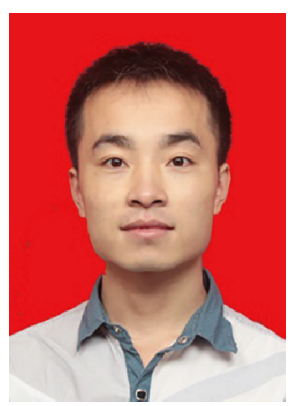

Yonghua Tang is a graduate student in Xiangtan University. He is currently studying at Peking University as an exchange student. His research is focused on the synthesis of nanostructures and their applications in photocatalytic degradation and hydrogen production.

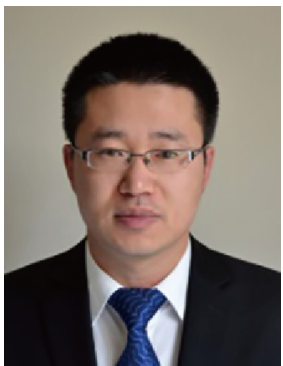

Shaojun Guo is currently a professor of materials science and engineering with a joint appointment at the Department of Energy\&Resources Engineering, the College of Engineering, Peking University. He received his BSc in chemistry from Jilin University (2005), $\mathrm{PhD}$ in analytical chemistry from Chinese Academy of Sciences (2010), worked as a postdoctoral research associate from Jan. 2011 to Jun. 2013 at Brown University and as a very prestigious J. Robert Oppenheimer Distinguished Fellow at Los Alamos National Laboratory. In 2014, 2015, 2016 and 2017, he was selected by Thomson Reuters into their prestigious list of World Most Highly Cited Researchers. His research interests are in engineering nanocrystals and 2D materials for catalysis, renewable energy, sensors and therapy.

\section{超薄钯纳米片/非晶氮化碳共面复合结构的控制生长及其可见光驱动下的光催化产氢性能研究}

唐永华 ${ }^{1,2}$, 周鹏 ${ }^{1}$, 沓玉 $广^{1}$, 林斐 ${ }^{1,3}$, 赖建平 ${ }^{1}$, 李红星 ${ }^{2}$, 郭少军 $^{1^{*}}$

摘要 目前, 能源与环境问题已经成为影响人类可持续发展的主要矛盾. 为了实现人类社会的可持续发展, 研究者们一直致力于开发新的 储能技术. 半导体光催化制氢凭借其清洁、可持续、环境友好的优势成为研究热点. 传统的光催化制氢体系以贵金属为助催化剂, 宽带隙 半导体为光催化剂, 这种光催化系统的太阳能转换效率难以满足实际需求. 在本文中, 我们合成出一种具有较大接触界面和较短的电子传 递路径的共面型超薄钯纳米片/非晶氮化碳复合结构. 在室温 $25^{\circ} \mathrm{C}$ 条件下, 该结构平均氢气生成速率为 $1.45 \mathrm{mmol} \mathrm{mg}^{-1} \mathrm{~h}^{-1}$, 是钯纳米颗粒无定形碳化氮粒面型结构的 2.6倍. 同时, 该共面型光催化剂具有优良的产氢稳定性. 该催化剂既充分利用了钯纳米片表面高密度的活性位 点, 又利用了无定型氮化碳宽的光谱响应. 本工作为可见光驱动的高效助催化剂和光催化剂界面设计提供了一种新的策略. 